\title{
AN ECONOMIC PREMIUM PRINCIPLE
}

\author{
HANS BÜHLMANN * \\ Eidgenössische Technische Hochschule, Zürich
}

1. PREMIUM CALCULATION PRINCIPLES VERSUS ECONOMIC PREMIUM PRINCIPLES

(a) The notion of premium calculation principle has become fairly generally accepted in the risk theory literature. For completeness we repeat, its definition:

A premium calculation principle is a functional $\mathfrak{S}$ assigning to a random variable $X$ (or its distribution function $F_{X}(x)$ ) a real number $P$. In symbols

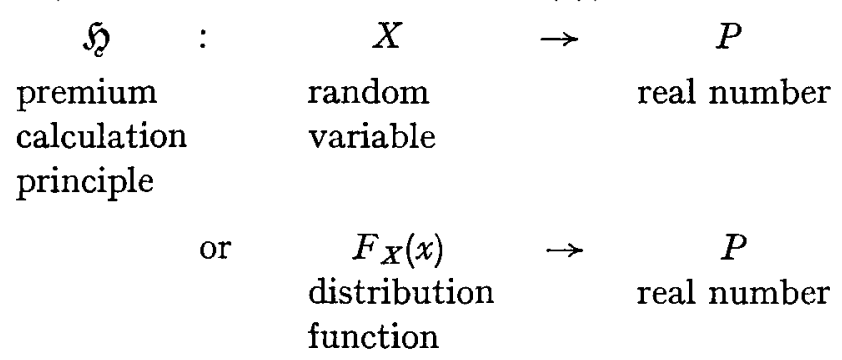

The interpretation is rather obvious. The random variable $X$ stands for the possible claims of a risk whereas $P$ is the premium charged for assuming this risk.

This is of course formalizing the way actuaries think about premiums. In actuarial terms, the premium is a property of the risk (and nothing else), e.g.

$$
\begin{aligned}
& \mathfrak{H}[X]=E[X]+\alpha \sigma[X] \\
& \mathfrak{H}[X]=(1+\lambda) E[X], \text { etc. }
\end{aligned}
$$

(b) Of course, in economics premiums are not only depending on the risk but also on market conditions. Let us assume for a moment that we can describe the risk by a random variable $X$ (as under a)), describe the market conditions by a random variable $Z$.

Then we want to show how an economic premium principle

$$
\begin{aligned}
& \text { F: } \quad(X, Z) \quad \rightarrow \quad P \\
& \text { pair of real number } \\
& \text { random } \\
& \text { variables }
\end{aligned}
$$

* This paper is greatly influenced by an exchange of ideas with Flavio Pressaco. I am also indebted to Hans Gerber for stimulating discussions on this subject. 
can be constructed. During the development of the paper we will also give a clear meaning to the random variable $Z$.

2. A MODEL FOR THE MARKET: RISK EXCHANGE

In the market we are considering agents $i=1,2, \ldots, n$. They constitute buyers of insurance, insurance companies, reinsurance companies.

Each agent $i$ is characterized by his utility function $u_{i}(x)$ [as usual: $u_{i}^{\prime}(x)>0, u_{i}^{\prime \prime}(x) \leqslant 0$ ] initial wealth $w_{i}$.

In this section, the risk aspect is modelled by a finite (for simplicity) probability space with states $s=1,2, \ldots, S$ and probabilities $\pi_{s}$ of state $s$ happening.

$$
\left(\sum_{i=1}^{s} \pi_{s}=1\right)
$$

\section{Terminology}

Each agent $i$ in the market has an original risk function $X_{i}(s)$ : payment caused to $i$ if $s$ is happening.

He is buying an exchange function $Y_{i}(s)$ : payment received by $i$ if $s$ is happening.

The notion of price for this purchase is given by a vector $\left(p_{1}, p_{2}, \ldots, p_{s}\right)=\mathrm{p}$ and the understanding that

$$
\text { Price }\left[Y_{i}\right]=\sum_{i-1}^{s} p_{s} Y_{i}(s)
$$

Hence $p_{s}$ is the price for one unit of conditional money (conditional upon the happening of state $s$ ).

\section{Definition}

$Y=\left(Y_{1}, Y_{2}, \ldots, Y_{n}\right)$ is a risk exchange $(\operatorname{REX})$ if $\sum_{i=1}^{n} Y_{i}(s)=0$ for all $s=1,2, \ldots, S$.

\section{Remark}

This condition represents the clearing condition of the market, which must be satisfied in any closed system.

\section{EQUILIBRIUM AND EQUILIBRIUM PRICE}

\section{Definition}

$(\mathbf{p}, \mathbf{Y})$ is called an equilibrium of the market if 
a) for all $i, \sum_{i=1}^{s} \pi_{s} u_{i}\left[w_{i}-X_{i}(s)+\mathbf{Y}_{i}(s)-\Sigma \mathbf{p}_{s} \mathbf{Y}_{i}(s)\right]=$ max for all possible choices of the exchange function $Y_{i}$.

b) $\sum_{i=1}^{n} \mathbf{Y}_{i}(s)=0$ for all $s=1,2, \ldots, S$.

\section{Terminology}

If conditions a) and b) are satisfied

$\mathbf{p}$ is called an equilibrium price,

$\mathbf{Y}$ is called an equilibrium REX.

The following observation, made in the form of a theorem is useful.

\section{Theorem $A$}

Condition a) in the definition of an equilibrium is satisfied if and only if

$$
\text { c) } \begin{aligned}
\frac{\pi_{t}}{\mathbf{p}_{i}} u_{i}^{\prime}\left[w_{i}-X_{i}(t)+\mathbf{Y}_{i}(t)\right. & \left.-\sum_{j=1}^{s} \mathbf{p}_{j} \mathbf{Y}_{i}(j)\right]= \\
& =\sum_{i=1}^{s} \pi_{s} u_{i}^{\prime}\left[w_{i}-X_{i}(s)+\mathbf{Y}_{i}(s)-\Sigma \mathbf{p}_{j} \mathbf{Y}_{i}(j)\right]
\end{aligned}
$$

holds for all $t$.

The proof of this theorem is obtained by partial differentiation for the "only if" part and follows from the concavity of $u_{i}(x)$ for the "if" part.

As a Corollary we see from this theorem that for any equilibrium price $\mathbf{p}$ we must have

$$
\sum_{i-1}^{s} \mathbf{p}_{s}=1
$$

Finally we must remember that an equilibrium as defined in this section always exists (see e.g. DeBREU [1] or [2]).

\section{THE CASE OF AN ARBITRARY PROBABILITY SPACE UNDERLYING THE RISK ASPECT}

The condition that the risk functions $X_{i}(s)$ and the exchange functions $Y_{i}(s)$ are random variables on a finite probability space is rather unnatural. We prefer to understand them as random variables $X_{i}(\omega), Y_{i}(\omega)$ on an arbitrary probability space $(\Omega, \mathfrak{A}, \Pi)$.

In this situation the notion of price is given by a function $\varphi: \Omega \rightarrow \mathbb{R}$, and the understanding that Price $\left[Y_{i}\right]=\int_{\Omega} Y_{i}(\omega) \varphi(\omega) d \Pi(\omega)$. 
We then define an equilibrium as the "pair" $(\boldsymbol{\varphi}, \mathbf{Y})$ such that

$\left.\mathrm{a}^{\prime}\right)$ for all $i$

$\int u_{i}\left[w_{i}-X_{i}(\omega)+\mathbf{Y}_{i}(\omega)-\int \mathbf{Y}_{i}\left(\omega^{\prime}\right) \boldsymbol{\varphi}\left(\omega^{\prime}\right) d \Pi\left(\omega^{\prime}\right)\right] d \Pi(\omega)=\max$ for all possible choices of the exchange function $Y_{i}(\omega)$.

b) $\sum_{i=1}^{n} \mathbf{Y}_{i}(\omega)=0 \quad$ for all $\omega$

\section{Remark}

The existence of such an equilibrium can not be proved in the same way as in the case of a finite probability space. Actually it is not clear whether an equilibrium always exists. In this paper, however, we take the pragmatic point of view of assuming existence. At least in the special case treated from section 5 on, this assumption turns out to be correct since we can explicitely calculate $\varphi$ and $\mathbf{Y}$.

The analogous theorem to $\mathrm{A}$ in section 3 then is the following:

\section{Theorem $A^{\prime}$}

Condition $\left.\mathrm{a}^{\prime}\right)$ in the definition of an equilibrium is satisfied if and only if

$\left.\mathrm{c}^{\prime}\right) u_{i}^{\prime}\left[w_{i}-X_{i}(\omega)+\mathbf{Y}_{i}(\omega)-\int \mathbf{Y}_{i}\left(\omega^{\prime}\right) \varphi\left(\omega^{\prime}\right) d \Pi\left(\omega^{\prime}\right)\right]$

$$
=\boldsymbol{\varphi}(\omega) \int u_{i}^{\prime}\left[w_{i}-X_{i}(\omega)+\mathbf{Y}_{i}(\omega)-\int \mathbf{Y}_{i}\left(\omega^{\prime}\right) \boldsymbol{\varphi}\left(\omega^{\prime}\right) d \Pi\left(\omega^{\prime}\right)\right] d \Pi(\omega)=C_{i} \boldsymbol{\varphi}(\omega)
$$

for almost all $\omega$.

\section{Remark 1}

Observe that $c^{\prime}$ ) is Borch's [3] condition characterizing a Pareto optimal risk exchange. Hence we have as a consequence of theorem $\mathrm{A}^{\prime}$ the

\section{Corollary}

A price equilibrium is a Pareto optimal risk exchange.

\section{Remark 2}

The converse of the corollary only holds if one allows for additional deterministic transfer payments.

\section{Proof of Theorem $A^{\prime}$}

(i) if part: First observe that under condition c') we have $\int \varphi(\omega) d \Pi(\omega)=1$

$$
\begin{aligned}
u_{i}\left[w_{i}-\right. & \left.X_{i}(\omega)+Y_{i}(\omega)-\int Y_{i}\left(\omega^{\prime}\right) \boldsymbol{\varphi}\left(\omega^{\prime}\right) d \Pi\left(\omega^{\prime}\right)\right] \\
\leqslant & u_{i}\left[w_{i}-X_{i}(\omega)+\mathbf{Y}_{i}(\omega)-\int \mathbf{Y}_{i}\left(\omega^{\prime}\right) \boldsymbol{\varphi}\left(\omega^{\prime}\right) d \Pi\left(\omega^{\prime}\right)\right] \\
& +u_{i}^{\prime}\left[w_{i}-X_{i}(\omega)+\mathbf{Y}_{i}(\omega)-\int \mathbf{Y}_{i}\left(\omega^{\prime}\right) \boldsymbol{\varphi}\left(\omega^{\prime}\right) d \Pi\left(\omega^{\prime}\right)\right] \\
& \times\left[Y_{i}(\omega)-\mathbf{Y}_{i}(\omega)-\int\left[Y_{i}\left(\omega^{\prime}\right)-\mathbf{Y}_{i}\left(\omega^{\prime}\right)\right] \boldsymbol{\varphi}\left(\omega^{\prime}\right) d \Pi\left(\omega^{\prime}\right)\right]
\end{aligned}
$$


The last summand can be rewritten using $\left.\mathrm{c}^{\prime}\right)$ as

$$
C_{i} \boldsymbol{\varphi}(\omega)\left[Y_{i}(\omega)-\mathbf{Y}_{i}(\omega)-\int\left[Y_{i}\left(\omega^{\prime}\right)-\mathbf{Y}_{i}\left(\omega^{\prime}\right)\right] \boldsymbol{\varphi}\left(\omega^{\prime}\right) d \Pi\left(\omega^{\prime}\right)\right]
$$

Integrating this term with $d \Pi(\omega)$ and using the fact that $\int \varphi(\omega) d \Pi(\omega)=1$ we obtain zero which completes the proof.

(ii) only if part: Choose an arbitrary measurable set $\mathrm{A}$ and a real number $\varepsilon$, and replace $\mathbf{Y}_{i}(\omega)$ by $\mathbf{Y}_{i}(\omega)+\varepsilon I_{A}(\omega)$

$$
\begin{aligned}
g(\varepsilon)=\int u_{i}\left[w_{i}-\right. & X_{i}(\omega)+\mathbf{Y}_{i}(\omega)+\varepsilon I_{A}(\omega)- \\
& \left.-\int\left\{\mathbf{Y}_{i}\left(\omega^{\prime}\right)+\varepsilon I_{A}\left(\omega^{\prime}\right)\right\} \boldsymbol{\varphi}\left(\omega^{\prime}\right) d \Pi\left(\omega^{\prime}\right)\right] d \Pi(\omega)
\end{aligned}
$$

is a differentiable real function with maximum at $\varepsilon=0$.

Hence we must have

$$
\begin{aligned}
& 0=g^{\prime}(0)=\int \frac{u_{i}^{\prime}\left[w_{i}-X_{i}(\omega)+\mathbf{Y}_{i}(\omega)-\int \mathbf{Y}_{i}\left(\omega^{\prime}\right) \boldsymbol{\varphi}\left(\omega^{\prime}\right) d \Pi\left(\omega^{\prime}\right)\right]}{\mathbf{Z}(\omega)} \\
& \times\left[I_{A}(\omega)-\int I_{A}\left(\omega^{\prime}\right) \varphi\left(\omega^{\prime}\right) d \Pi\left(\omega^{\prime}\right)\right] d \Pi(\omega)
\end{aligned}
$$

Hence

$$
\int_{A} \mathbf{Z}(\omega) d \Pi(\omega)=\int_{A} \boldsymbol{\varphi}(\omega) d \Pi(\omega) \cdot \int \mathbf{Z}(\omega) d \Pi(\omega)
$$

Since this relation is true for any measurable set $A$, we must have

$$
\mathbf{Z}(\omega)=\boldsymbol{\varphi}(\omega) \int \mathbf{Z}(\omega) d \Pi(\omega) \quad \text { for almost all } \omega,
$$

which is condition $\left.c^{\prime}\right)$ in abbreviated notation.

5. THE CASE OF EXPONENTIAL UTILITY FUNCTIONS

We are now applying Theorem $A^{\prime}$ in the case of exponential utilities

$$
u_{i}(x)=\frac{1}{\alpha_{i}}\left[1-e^{-\alpha_{i} x}\right]
$$

where

$$
u_{i}^{\prime}(x)=e^{-\alpha_{i} x}
$$

$\alpha_{i}$ stands for the risk aversion,

$\frac{1}{\alpha_{i}}$ stands for the risk tolerance unit.

Condition $\mathrm{c}^{\prime}$ ) then becomes

(2) $\exp \left[-\alpha_{i}\left[w_{i}-X_{i}(\omega)+\mathbf{Y}_{i}(\omega)-\int \mathbf{Y}_{i}\left(\omega^{\prime}\right) \boldsymbol{\varphi}\left(\omega^{\prime}\right) d \Pi\left(\omega^{\prime}\right)\right]\right]=C_{i} \boldsymbol{\varphi}(\omega)$ a.s.

As any optimal $\mathbf{Y}_{i}(\omega)$ is only determined up to an additive constant, there is no loss of generality if we assume that

$$
\left.\mathrm{d}^{\prime}\right) \int \mathbf{Y}_{i}\left(\omega^{\prime}\right) \boldsymbol{\varphi}\left(\omega^{\prime}\right) d \Pi\left(\omega^{\prime}\right)=0 .
$$


Absorbing $e^{-\alpha_{i} w_{i}}$ into the constant $C_{i}$, which then becomes $\mathrm{C}_{i}$, we have

$$
\exp \left[\alpha_{i} X_{i}(\omega)-\alpha_{i} \mathbf{Y}_{i}(\omega)\right]=\mathrm{C}_{i} \varphi(\omega) \quad \text { a.s. }
$$

or

(3)

$$
X_{i}(\omega)-\mathbf{Y}_{i}(\omega)=\frac{1}{\alpha_{i}}\left[\ln \mathrm{C}_{i}+\ln \varphi(\omega)\right] \quad \text { a.s. }
$$

We now use the clearing condition $b^{\prime}$ )

$$
\sum_{i=1}^{n} \mathbf{Y}_{i}(\omega)=0 \quad \text { for all } \omega
$$

and the abbreviation $\sum_{i=1}^{n} X_{i}(\omega)=Z(\omega)$.

Summing equation (3) over $i$, we obtain

(4) $\quad Z(\omega)=\frac{1}{\alpha} \ln \varphi(\omega)+k$, where $\frac{1}{\alpha}=\sum_{i=1}^{n} \frac{1}{\alpha_{i}}$

$k$ a constant independent of $\omega$ and $i$

$$
\left(k=\sum_{i=1}^{n} \frac{1}{\alpha_{i}} \ln \mathrm{C}_{i}\right) .
$$

The last equation yields

$$
e^{\alpha Z} \boldsymbol{Z}(\omega)=e^{\alpha k} \varphi(\omega)
$$

and the condition $\int \varphi(\omega) d \Pi(\omega)=1$ finally leads to

$$
\varphi(\omega)=\frac{e^{\alpha Z(\omega)}}{E\left[e^{\alpha Z}\right]}
$$

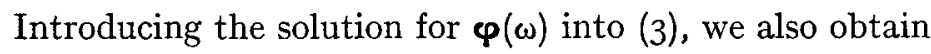

$$
\begin{aligned}
X_{i}(\omega)-Y_{i}(\omega) & =\frac{\frac{1}{\alpha_{i}}}{\frac{1}{\alpha}} Z+k_{i} \\
& =\gamma_{i} Z+k_{i}, \quad \text { where } \gamma_{i}=\frac{\frac{1}{\alpha_{i}}}{\frac{1}{\alpha}}
\end{aligned}
$$

and $\Sigma k_{i}=0$. 


\section{Remark}

From an easy exercise using BoRch's theorem [3] one finds that the "allocation after the exchange" which is described by equation (6) is Pareto optimal. As we know, this result, verified by explicit calculation in the special case of exponential utility functions, is generally true. (See corollary after Theorem $\mathrm{A}^{\prime}$ ).

Finally the constants $k_{i}$ appearing in (6) can be determined from $\int \mathbf{Y}_{i}(\omega) \boldsymbol{\varphi}(\omega) d \Pi(\omega)=$ o. Hence we have

$$
\int X_{i}(\omega) \boldsymbol{\varphi}(\omega) d \Pi(\omega)=\gamma_{i} \int Z(\omega) \boldsymbol{\varphi}(\omega) d \Pi(\omega)+k_{i}
$$

or

$$
\frac{E\left[X_{i} e^{\alpha Z}\right]}{E\left[e^{\alpha Z}\right]}=\gamma_{i} \frac{E\left[Z e^{\alpha Z}\right]}{E\left[e^{\alpha Z}\right]}+k_{i} \quad \text { for all } i
$$

Hence

$$
\mathbf{Y}_{i}(\omega)=X_{i}(\omega)-\gamma_{i} Z(\omega)-\frac{E\left[\left(X_{i}-\gamma_{i} Z\right) e^{\alpha} Z\right]}{E\left[e^{\alpha Z}\right]}
$$

Observe that our explicitely calculated $(\boldsymbol{\varphi}, \mathbf{Y})$ is an equilibrium which a posteriori justifies our original assumption of existence of such equilibrium.

\section{AN ECONOMIC PREMIUM PRINCIPLE}

Equation (5) defines an economic premium principle for all random variables $X: \Omega \rightarrow \mathbb{R}$. We have

$$
\mathfrak{F}_{\alpha}[X, Z]=\frac{E\left[X e^{\alpha Z}\right]}{E\left[e^{\alpha Z}\right]} \text { where } Z=\sum_{i=1}^{n} X_{i} \text { is the sum of original risk }
$$
functions in the market.

It is most interesting to observe that in the case where $X$ and $Z-X$ are independent, one obtains

$$
\xi_{\alpha}[X, Z]=\frac{E\left[X e^{\alpha X}\right] E\left[e^{\alpha(Z-X)}\right]}{E\left[e^{\alpha X}\right] E\left[e^{\alpha(Z-X)}\right]}=\frac{E\left[X e^{\alpha X}\right]}{E\left[e^{\alpha X}\right]} .
$$

Hence in this case our economic principle reduces to a "standard" premium calculation principle

$$
\begin{aligned}
\mathfrak{H}_{\alpha}[X]=\frac{E\left[X e^{\alpha X}\right]}{E\left[e^{\alpha X}\right]}, \begin{array}{l}
\text { which one might call the Esscher principle (be- } \\
\text { cause of its formal connection with the Esscher } \\
\text { transform). }
\end{array}
\end{aligned}
$$


The parameter $\frac{1}{\alpha}$, as we have seen, has the intuitive meaning of the risk tolerance unit of the whole market.

Let us compare this (new) Esscher principle with the exponential principle for agent $i$ (with risk tolerance unit $\frac{1}{\alpha_{i}}$ )

$$
\mathfrak{H}_{\alpha_{i}}^{(i)}[X]=\frac{1}{\alpha_{i}} \ln E\left[e^{\alpha_{i} X}\right] .
$$

The two principles can also be written by means of the moment generating function $M_{X}(t)=E\left[e^{t X}\right]$ and its derivative $M_{X}^{\prime}(t)=E\left[X e^{t X}\right]$.

Hence

$$
\begin{gathered}
\mathfrak{H}_{\alpha}[X]=\frac{M_{X}^{\prime}(\alpha)}{M_{X}(\alpha)}=\left.\frac{d}{d t}\left[\ln M_{X}(t)\right]\right|_{t=\alpha} \\
\mathfrak{W}_{\alpha_{i}}^{(i)}[X]=\frac{1}{\alpha_{i}} \ln M_{X}\left(\alpha_{i}\right) .
\end{gathered}
$$

Observe that $\mathfrak{J}_{\alpha}[X] \uparrow$ as $\alpha \uparrow$ and hence

$$
\mathfrak{H}_{\beta}^{(i)}[X]=\frac{1}{\beta} \int_{0}^{\beta} \frac{M_{X}^{\prime}(t)}{M_{X}(t)} d t \leqslant \frac{M_{X}^{\prime}(\beta)}{M_{X}(\beta)}=\mathfrak{H}_{\beta}[X] .
$$

Hence

$$
\mathfrak{H}_{\beta}^{(i)}[X] \leqslant \mathfrak{T}_{\beta}[X]
$$

and approximately

$$
\mathfrak{H}_{\beta}^{(i)}[X] \sim \mathfrak{H}_{\bar{\beta}}[X]
$$

Formulae (9) and (10) give rise to an interesting interpretation.

A newcomer (numbered as $n+1$ ) to the market, who only offers cover but does not bring any additional risks (e.g. a professional reinsurer) can easily check individual rationality by testing whether

$$
\mathfrak{H}_{\mathcal{g} \alpha_{n+1}}^{(i)}[X] \leqslant \mathfrak{H}_{\alpha}[X], \quad \text { where } \quad \frac{1}{\alpha}=\sum_{i=1}^{n+1} \frac{1}{\alpha_{i}}
$$

According to (10), the bordercase for individual rationality is about characterized by

$$
\alpha_{n+1}=2 \alpha \text { hence } \frac{1}{\alpha_{n+1}}=\frac{1}{2}\left(\frac{1}{\alpha_{1}}+\ldots+\frac{1}{\alpha_{n}}\right)+\frac{1}{2} \frac{1}{\alpha_{n+1}}
$$




$$
\text { or } \frac{1}{\alpha_{n+1}}=\frac{1}{\alpha_{1}}+\ldots+\frac{1}{\alpha_{n}}
$$

which leads to the "rule of thumb" that the market should only be joined by such a newcomer if he offers at least as many new risk tolerance units as already present in the market before him.

\section{REFERENCES}

[1] Debreu, G. (1974). Four Aspects of the Mathematical Theory of Economic Equilibrium, Proceedings of the International Congress of Mathematicians, Vancouver.

[2] Debreu, G. (1976). Werttheorie (see Anhang), Springer Verlag.

[3] Borch, K. (1960). The Safety loading of Reinsurance Premiums, Skandinavisk Aktuarietidskrift. 